\title{
Time to activate!
}

Align Technology, Inc. (NASDAQ:

ALGN) has announced the introduction of Invisalign G8 with SmartForce Aligner Activation, the latest of the company's biomechanics innovations.

Invisalign G8 with SmartForce Aligner Activation is informed by the company's foundational biomechanics for clear aligners and its database of more than 9 million Invisalign patients to optimise tooth movements and further improve predictability for frequently treated crowding, crossbite, and deep bite cases.

With SmartForce Aligner Activation, select areas of the aligner surface are specifically contoured to apply optimal forces to the tooth surfaces to control the location, direction and intensity of the force to produce the desired outcome and minimise unwanted movement. Specific, strategic contact areas between the aligner and the tooth are created by SmartForce Aligner Activation and work in concert with SmartForce features for even greater control of the force systems.

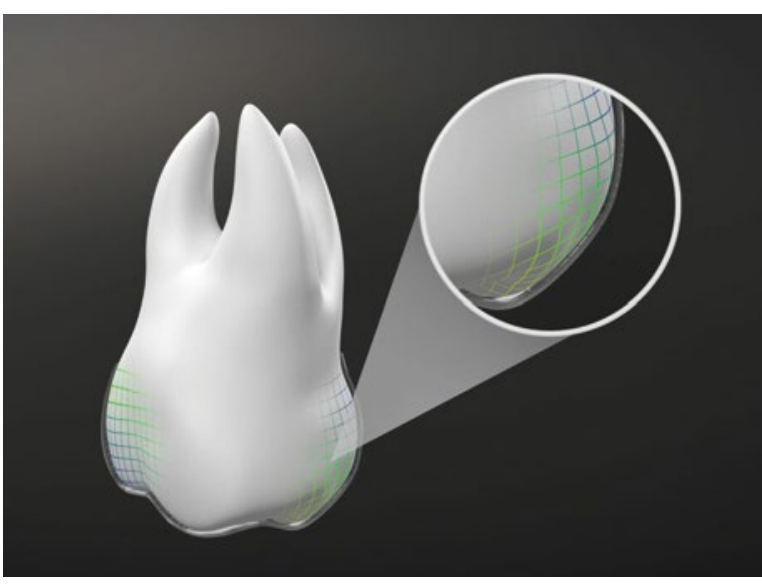

Invisalign G8 with new SmartForce Aligner Activation ensures sufficient and consistent activation in every aligner stage to help doctors get more of the desired movements from every aligner in the treatment of crowding, crossbite, and deep bite.

\section{For crowding and crossbite cases:}

Smart Force Aligner Activation aids in posterior arch expansion by working synergistically with New Optimised Expansion Support attachments or Optimised Expansion Support and Rotation attachments to reduce the potential for buccal crown tipping during posterior arch expansion.

\section{For deep bite cases:}

SmartForce Aligner Activation supports anterior intrusion with improvements in the treatment plan set-ups to level the curve of speed and demonstrates up to $2 \mathrm{x}$ improvement in predictability of incisor intrusion.

In addition, with Invisalign G8, doctors can now select automatic placement of Precision Bite Ramps during the prescription process. Data demonstrate that Precision Bite Ramps improve lower intrusion in deep bite cases. Invisalign G8 will be available globally on all Invisalign products in the first quarter of 2021. For more information visit www.aligntech.com.

\section{Oral hygiene - more}

\section{than just brushing}

For patients to maintain optimal oral hygiene at home, it takes more than just brushing their teeth. Why not introduce them to the Curasept ADS range of mouth rinses to take their dental health to the next level?

Available from J\&S Davis, Curasept ADS products contain chlorhexidine for plaque fighting benefits, while being formulated to minimise the risk of staining and taste disturbance associated with other solutions. There are also targeted therapies like Curasept ADS Implant and Curasept ADS Perio, which include hyaluronic acid to accelerate healing and PVP-VA to combat future plaque accumulation.

To help your patients elevate their homecare dental routines, remind them it takes more than just brushing.

For more information on the industry-leading products available from J\&S Davis, visit www.js-davis. co.uk, call 01438747344 or email jsdsales@js-davis.co.uk.
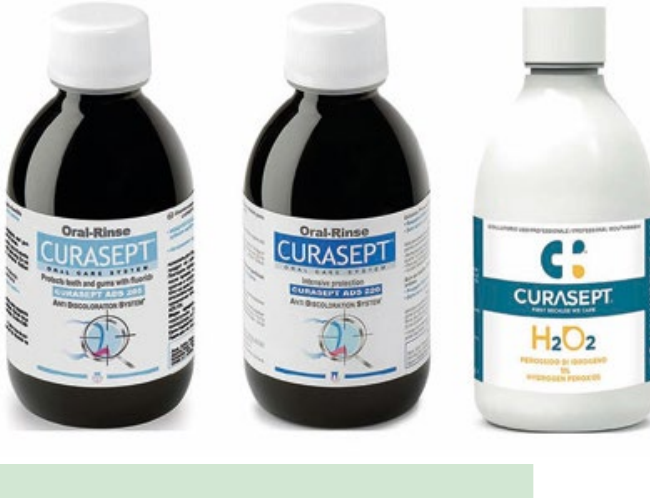

\section{Small but mighty}

Are you looking for a cutting-edge piece of CBCT technology but worried that size may be an issue?

Never compromise and open a whole new world of imaging with the CS 8100 3D Evo Edition from Carestream Dental.

Versatile to your needs and compact, the system features an extended field of view that opens the door for practices looking to expand their treatment capabilities.
Furthermore, as the system is a 4 -in-1 solution, professionals can easily shift between cephalometric, panoramic, extraoral bitewing imaging and more, meaning that the system is perfect for even advanced treatments such as orthodontics and dental implant planning.

For more information, contact Carestream Dental on 08001699692 or visit www.carestreamdental.co.uk.

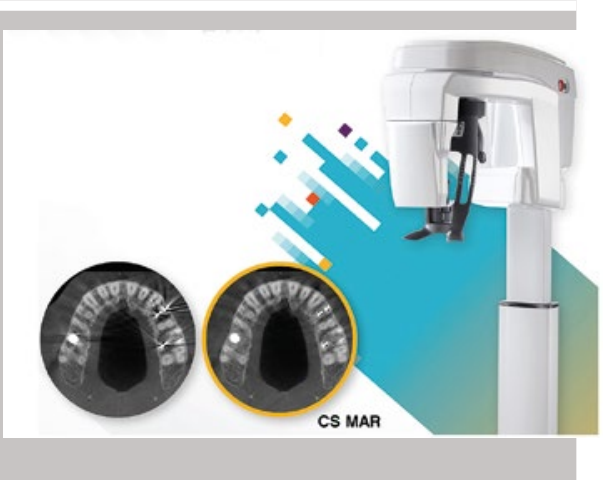

